\title{
Gaze Guidance Using a Facial Expression Robot
}

\author{
Minoru Hashimoto ${ }^{\mathrm{a}, *}$, Hiromi Kondo $^{\mathrm{b}}$ and Yukimasa Tamatsu ${ }^{\mathrm{c}}$ \\ ${ }^{a}$ Department of Bioscience and Textile Technology, Interdisciplinary Graduate School of Science \\ and Technology, Shinshu University, 3-15-1 Tokida, Ueda, Nagano 386-8567, Japan \\ ${ }^{\mathrm{b}}$ Hayashi Telempu Co. Ltd., 100-11 Machiyabora, Kamekubi, Toyota, Aichi 470-0375, Japan \\ ${ }^{c}$ DENSO Co. Ltd., 1-1 Shouwa, Kariya, Aichi 448-8661, Japan
}

Received 7 July 2009; accepted 8 July 2009

\begin{abstract}
This paper describes the gaze guidance with emotional expression of a head robot, called Kamin-FA1. We propose to use not only the gaze control of the robot, but also the facial expression to guide a human being's gaze to the target. We provide the information of the target of gaze intuitively to the human based on joint attention with Kamin-FA1. The robot has a facial expression function using a curved surface display. We examined the effect of emotional expression on the gaze guidance in terms of the accuracy and reaction speed. We conducted experiments of human gaze measurement during the gaze guidance with emotional expression to evaluate the role of emotional expression. The results of the gaze guidance experiments showed that gaze guidance with emotional expression caused a more accurate and quicker response than that without emotional expression. In particular, the expression of surprise has better performance in the gaze guidance compared with the normal expression. Furthermore, emotional expressions of angry and surprise impressed the subjects in dangerous situations, while normal and happy situations gave the impression of a safe situation at the target of gaze.

(c) Koninklijke Brill NV, Leiden and The Robotics Society of Japan, 2009
\end{abstract}

\section{Keywords}

Communication robot, gaze guidance, facial expression, joint attention, emotion

\section{Introduction}

Recently, robots purposely designed for communicating with humans [1, 2] have attracted our attention. The ability to communicate is indispensable for humanrobot symbiosis. It is well known that facial expressions play a very important role in daily communication. In order to familiarize the robot with human society, it is essential to create an affinity with facial expressions. From this perspective, we developed a head robot called Kamin-FA1 (KAnsei MINd robot) [3] to enhance

\footnotetext{
* To whom correspondence should be addressed. E-mail: hashi@ shinshu-u.ac.jp
} 
emotional communication with humans. This robot has a facial expression function using a curved surface display as well as head motion using a neck mechanism with 4 d.o.f. We applied the robot to a human-robot Kansei communication based on emotional synchronization [4].

On the other hand, many studies of intelligent transport systems (ITS) have been conducted. Much information, such as car environment information and physiological information of the driver, can be provided by the progress of ITS. However, there has been no consideration about the interface to present information to the driver intuitively by such means as facial expression or sound. This means that the driver is required to constantly pay attention to observe information. Thus, it is important to make a human-machine interface for safe and comfortable driving conditions. Recently, a robotic car agent [5] was proposed to identify key interface design issues through pilot testing. It is necessary to study the communication technique between the car robot and the driver to realize safe driving conditions.

One of the important roles of the interface is gaze guidance for the driver to recognize the situation of the car. We can provide the information of the gaze target intuitively and easily to the driver based on joint attention with the robot. Therefore, gaze guidance based on joint attention is one of the communication techniques for robots. Generally, our gaze movement involves emotional expressions. For example, if a pedestrian appears in front of a car suddenly, we exhibit an expression of fear or surprise. In this case we think that the emotional expression helps to communicate what is going on in front of the car and urge the driver to make a quick response.

There are some studies on finding the relationship between facial expression and eye gaze. Putman et al. [6] found that fearful faces induced stronger gaze cuing than happy faces and the strength of this cuing effect was correlated with the participants' anxiety level. Using synthetic dynamic facial expressions, Sander et al. [7] showed that angry faces are recognized as expressing more anger and the underlying emotion is judged more intensively with a direct than with an averted gaze. These findings show that there are interactions between cognitive processes of gaze cuing and emotional expression, and the strength of interaction depends on the kinds of emotion. In other literature [8-10] it was shown that the change of emotional expression is an important influence on cuing.

From this point of view, we investigate gaze guidance using the head robot Kamin-FA1. In this paper the phrase 'gaze guidance' means that the human gaze is guided by another expression including eye and face movement with the facial expression. We use not only gaze control of the robot, but also facial expressions to guide the human gaze to the desired target. We examine the role of the emotional expression for gaze guidance and reaction speed during the gaze guidance with emotional expressions. The differences among the emotional expressions in terms of the effectiveness of gaze guidance using the robot were investigated. The performance of gaze guidance is examined by the maximum gaze angle and the delay time of human gaze movement. Also, we measure the reaction speed of an 
operation with gaze guidance. We need some operations after recognizing a dangerous situation to avoid the accident. Then, we investigate the role of emotional expression in terms of the reaction speed of the operation.

There have been many studies on joint attention between humans and robots (e.g., Refs [11, 12]). The aims of these studies concern the gaze control of the robot to establish joint attention. There have been few studies on gaze guidance of humans interacting with a robot. Osawa et al. [13] proposed a display robot to guide human gaze to the target. However, they used only gaze control of the display robot in the gaze guidance. There has been no study on the effect of the emotional expression on the gaze guidance.

In Section 2, the emotional expression and gaze control of the head robot KaminFA1 are described in more detail. Also, the principle of the curved surface display and the neck mechanism are explained. In Section 3, we conduct experiments on the gaze guidance of subjects by using the emotional expression of Kamin-FA1. In Section 4 , the reaction speed after gaze guidance is measured. Also, psychological experiments are performed to examine its validity in terms of the presentation of situations at the gaze target in Section 5. Finally, in Section 6, we conclude this paper by describing the possible applications in automobiles for a human-machine interface.

\section{Emotional Expression and Gaze Control}

\subsection{Outline of Kamin-FA1}

The structure and overview of Kamin-FA1 is shown in Fig. 1. The system consists mainly of head and neck mechanisms. The head part is a facial image display, and
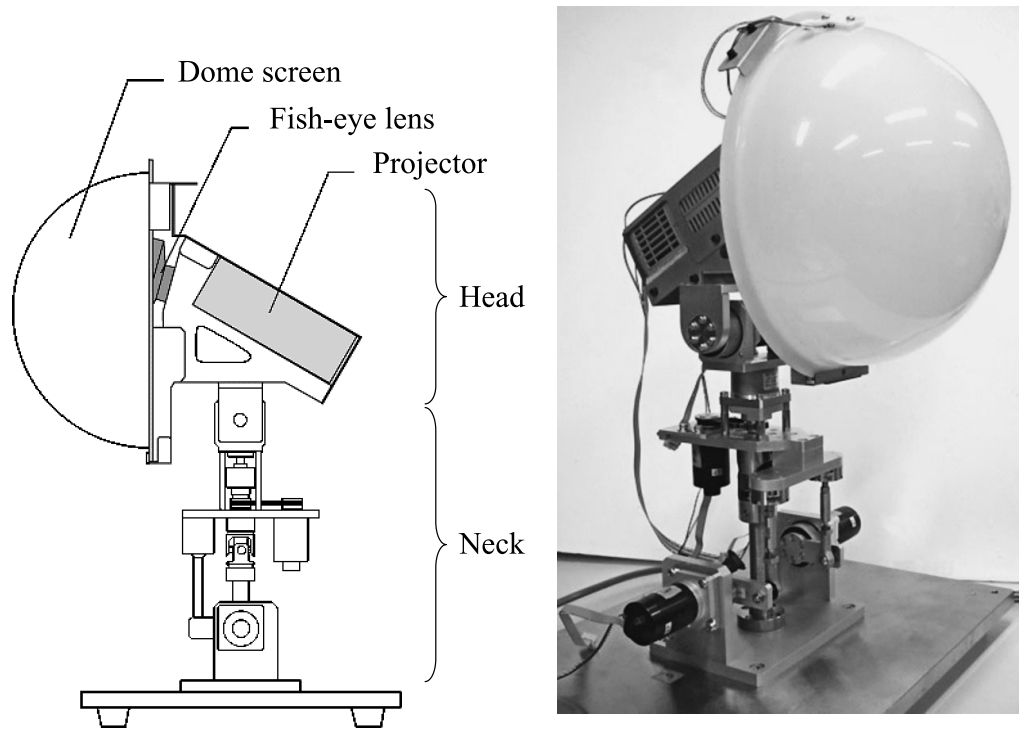

Figure 1. Structure and overview of Kamin-FA1. 

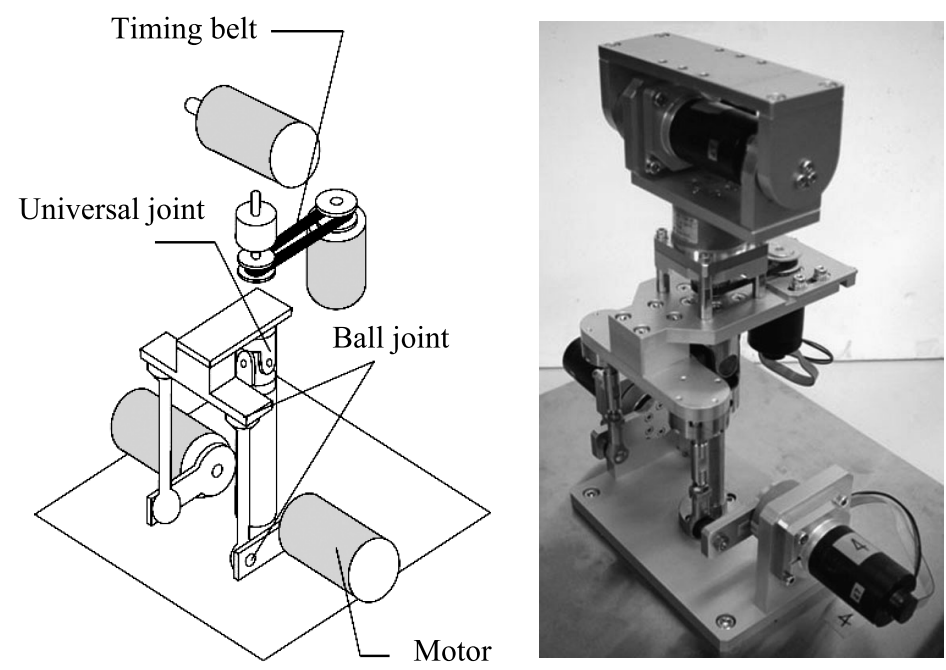

Figure 2. Structure and overview of the neck mechanism of Kamin-FA1.

consists of a dome screen, a fish-eye lens and a projector. The dome screen is a milky white translucent acrylic dome and it is considered as the face of the robot's head. The face image is projected on the dome screen from the inside. The fish-eye lens is installed on the front of the projector and it projects the picture on the dome screen. By using the fish-eye lens, the image can be projected onto the surface of the sphere. A digital light processing projector, V-807 (PLUS-Vision), is used. The projector is very compact (width $180 \mathrm{~mm}$, height $45 \mathrm{~mm}$ and diameter $141 \mathrm{~mm}$ ) and light $(0.9 \mathrm{~kg})$. The diameter of the dome screen is $250 \mathrm{~mm}$. The visual field of the fish-eye lens (Nikon, FC-E8) is about $180^{\circ}$. The head is put in a sphere of $300 \mathrm{~mm}$ in diameter and it weighs approximately $2.5 \mathrm{~kg}$.

The motion of the head part is performed by a neck mechanism. Figure 2 shows the structure and overview of the neck mechanism. The neck mechanism has 4 d.o.f., and consists of four motors, a timing belt and a parallel linkage mechanism. Using a belt for the second motor shortens the vertical length of the neck mechanism. Ball joints are used as the joints in a parallel linkage mechanism, and a universal joint is used as the center of inclination for the head in the direction of front and rear, right and left. The head inclines forward and backward when two lower motors rotate in the same directions, and inclines to the right and left when they rotate in different directions. Since two motors are placed on the base, the operating loads for the motors are reduced.

Four 25-W DC motors (Faulhaber, 3242G024CR) were used. A harmonic drive gear is attached to each motor. The reduction ratio is $1 / 100$. The distance between the universal joint and the rotational axis of the top motor is $120 \mathrm{~mm}$ and the total height of the neck mechanism is about $300 \mathrm{~mm}$. The rotational ranges are $\pm 30^{\circ}$ in tilt rotation, $30^{\circ}$ in forward rotation, $70^{\circ}$ in backward rotation and $\pm 90^{\circ}$ in horizontal rotation. The total weight of the neck mechanism is $3.5 \mathrm{~kg}$. 


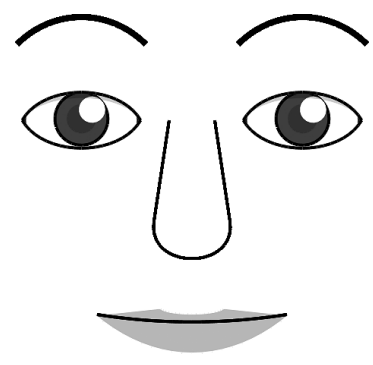

(a) Face image

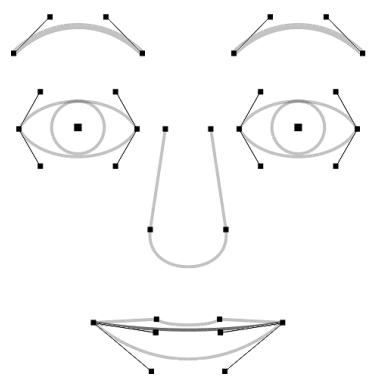

(b) Face model

Figure 3. Face image and its model.

\subsection{Drawing the Face Image}

Figure 3 shows an example of the face image and its model. The face image is made up of a line drawing and it is determined numerically. The face image consists of straight lines, bezier curves and circles. A straight line can be created by specifying two points. Similarly, a bezier curve is four points, and a circle is the center point and a radius. That means the state of facial expressions is specified with the parameter vector of the points and the radii.

The projected image on the dome screen has observation direction dependability. Thus, we can feel the three-dimensional effect on the face model. Also, each part of the face model can be moved very easily with a fast response because the face model is only animated computer graphics.

\subsection{Emotional Expression}

An emotional state is expressed within the parameters of six basic emotions: happiness, sadness, anger, fear, surprise and disgust. The parameter vector of the facial image is computed from this emotional state. The expression model of the emotional state is made by the Action Unit of the Facial Action Coding System as proposed by Ekman and Friesen [14]. Using this method, subtle expressions between emotional states are possible by using the parameter vector.

We evaluated the impression of facial expressions by psychological experiments. We presented nine kinds of face images as shown in Fig. 4 to the subjects and asked their impression in terms of emotions such as angry, surprise, etc. The number of the subjects was 20 .

Table 1 lists the results of the impression evaluation. We selected the facial expressions of the basic six emotions based on the results. Sample 1 is used as a face image for angry, Sample 2 is for surprise, Sample 7 is for happy, Sample 4 is for fear, Sample 5 is for disgust and Sample 6 is for sad. The score of Sample 3 is as high as a face image for happy; however, the pupil was not shown in the face image. Therefore, we selected Sample 7 as a face image for happy. We can feel the intentional emotion from these facial images. There are differences of eye visibility 


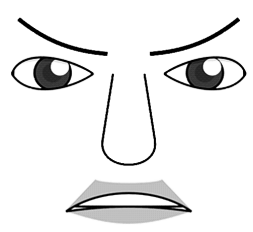

(a) Sample 1

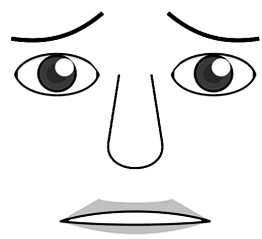

(d) Sample4

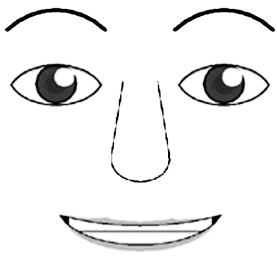

(g) Sample7

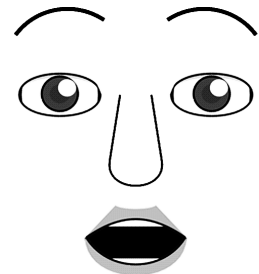

(b) Sample2

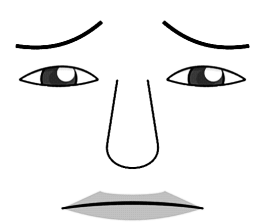

(e) Sample5

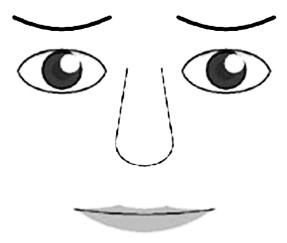

(h) Sample8

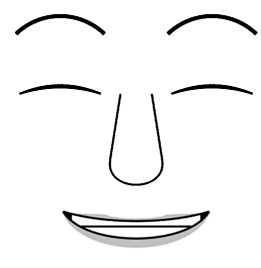

(c) Sample3

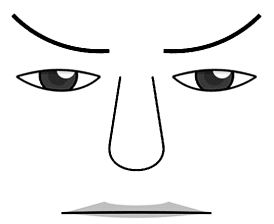

(f) Sample6

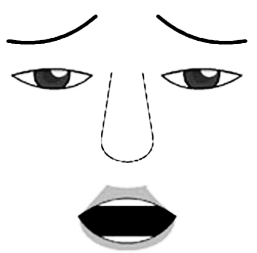

(i) Sample9

Figure 4. Facial expressions of Kamin-FA1.

Table 1.

Result of impression evaluation

\begin{tabular}{llllllll}
\hline \multirow{2}{*}{ Sample } & Answers & \multicolumn{7}{l}{} \\
\cline { 2 - 8 } & Angry & Surprise & Happy & Fear & Disgust & Sad & Others \\
\hline 1 & 20 & - & - & - & - & - & - \\
2 & - & 20 & - & - & - & - & - \\
3 & - & - & 20 & - & - & - & - \\
4 & - & - & - & 17 & 2 & 1 & - \\
5 & 3 & - & - & 2 & 14 & - & 1 \\
6 & - & - & - & - & 4 & 15 & 1 \\
7 & - & - & 18 & - & - & - & 2 \\
8 & - & - & - & 4 & 4 & 9 & 2 \\
9 & - & - & - & 7 & 4 & 9 & - \\
\hline
\end{tabular}

between emotional expressions. These differences of eye visibility could affect the gaze guidance. 


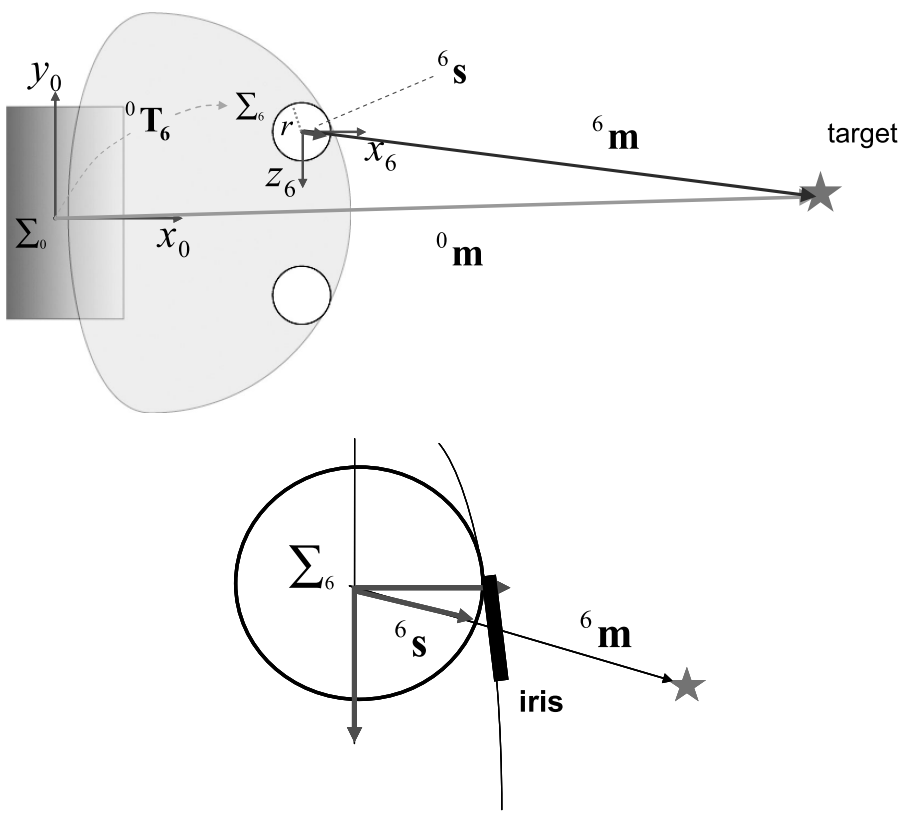

Figure 5. Kinematics for gaze control.

\subsection{Gaze Control}

The eye movement of Kamin-FA1 is controlled so that the gaze of the robot directs the human gaze to the target. To determine the iris positions on the robot's face, we computed the kinematics of the neck mechanism. Figure 5 shows the reference and eye coordinate fames as well as the target point. The frame $\Sigma_{0}$ is the reference coordinate fixed on the base. The frame $\Sigma_{6}$ is an eye coordinate fixed on the head. Using the homogeneous transformations we can calculate the vector of the target point relative to $\Sigma_{6},{ }^{6} \mathbf{m}$, by:

$$
{ }^{6} \mathbf{m}={ }^{6} \mathbf{T}_{0}{ }^{0} \mathbf{m},
$$

where ${ }^{0} \mathbf{m}$ is the position vector of the target point relative to $\Sigma_{0}$ and ${ }^{6} \mathbf{T}_{0}$ is the homogeneous transformation matrix from $\Sigma_{6}$ to $\Sigma_{0}$. Then, the intersection between the eye ball and the target vector ${ }^{6} \mathbf{m}$ is determined by:

$$
{ }^{6} \mathbf{s}=\frac{r}{\left|{ }^{6} \mathbf{m}\right|}{ }^{6} \mathbf{m}
$$

where ${ }^{6} \mathbf{s}$ is the position vector of the intersection relative to $\Sigma_{6}$ and $r$ is the radius of the eye ball. Since the intersection ${ }^{6} \mathbf{s}$ is not on the screen surface, we determined the position of the iris on the screen surface using the $y$ and $z$ components of the intersection vector, ${ }^{6} \mathbf{s}$. The eye movement is performed in real-time using the joint angles of the neck mechanism. 


\section{Gaze Guidance with Emotional Expression}

We have conducted experiments to investigate the effectiveness of gaze control and emotional expressions to gaze guidance. Human beings have the ability of joint attention; in other words, our gaze entrains others to gaze. Thus, if we control the robot gaze to the target, a human being's gaze can be guided to the target. Figure 6 shows the experimental setup. The subject wearing an eye mark recorder sat down in front of the robot. The eye mark recorder that we used is EMR-8B made by nac Image Technology Inc. The resolution of this eye mark recorder is $0.1^{\circ}$. The sampling rate is $30 \mathrm{~Hz}$. The subject interacted with the robot for a few minutes. When the robot's gaze changed from the subject position to another position with facial expression, we measured the subject's gaze by using the eye mark recorder to examine the validity of the gaze guidance.

The robot's gaze was controlled to move from the subject to the left or right direction, stop for $1 \mathrm{~s}$ at $25^{\circ}$ and then return to the starting position. The speed of gaze movement is $25 \%$, and the distance between the robot and the subject is $1.5 \mathrm{~m}$ as shown in Fig. 7. The transition periods of the robot gaze movement and the facial expression are 0.17 and $0.21 \mathrm{~s}$, respectively (Fig. 8). The change speeds of the gaze and facial expression are faster than that of the head motion. We applied six kinds of emotional expressions combining eye and head movements to the subjects. The emotional expressions consisted of happy, angry, surprise, sad, fear and disgust. The order of the emotions was randomized for each participant, so that any training/sequencing effects were canceled out. Examples of facial expressions used during the gaze guidance experience are shown in Fig. 9. The number of subjects was 30 . The participants were instructed simply to interact with the robot. We did not instruct them to follow the robot's gaze.

Examples of the gaze trajectories of subjects are shown in Fig. 10. The solid line shows the gaze trajectory of the subject and the broken line shows the gaze movements of Kamin-FA1 with various emotional expressions. The gaze trajectories of the subjects depend on the emotional expression of Kamin-FA1. The maximum

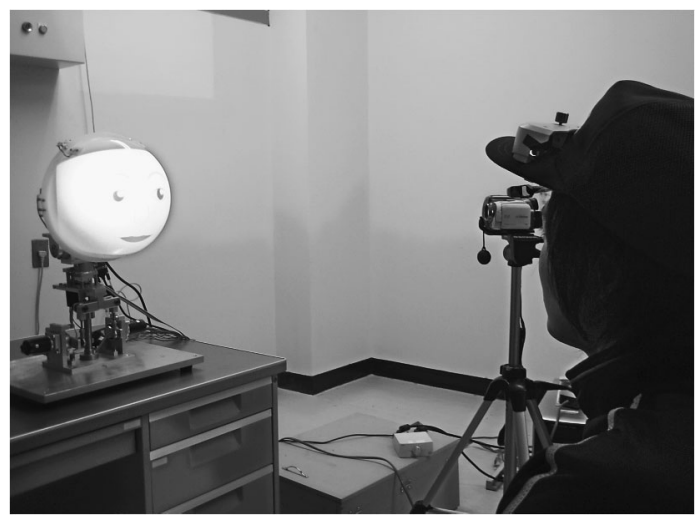

Figure 6. Overview of the gaze guidance experiment. 


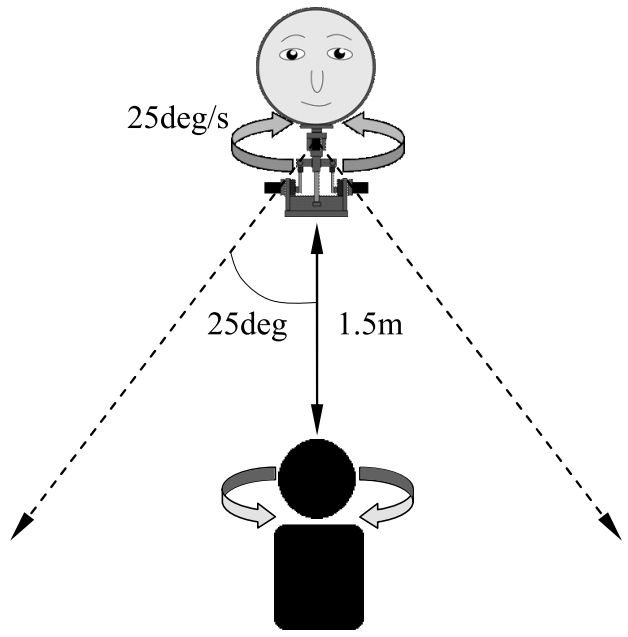

Figure 7. Schematic drawing of the gaze guidance experiment.

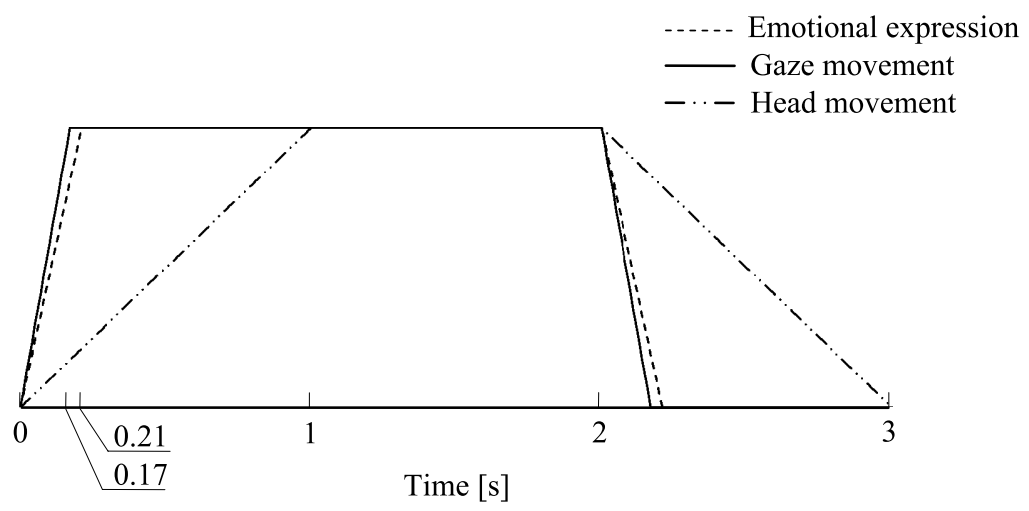

Figure 8. Change of gaze and emotional expression of Kamin-FA1.

gaze angle and the delay time of the subject are obtained from the gaze trajectories of the subjects as shown in Fig. 11. The maximum gaze angle is the maximum angle of the subject's gaze during the gaze guidance of Kamin-FA1. The time delay is determined by measuring the time between when the robot head reaches the $25^{\circ}$ angle and when the subject's gaze changes. We consider that if the maximum gaze angle is larger and the time delay is shorter, then the performance of the gaze guidance is better in terms of accuracy and reaction speed. The deviation of the robot's gaze is $25^{\circ}$, but the subject's gaze angle was not $25^{\circ}$. The subject was directed to the robotic line of sight, since something may have happened to warrant checking. However, when no object was observed along the line, the subject's line of sight returned to the original position. We can understand if there is something to observe or not from the peripheral field of view. If the emotional expression impresses the 

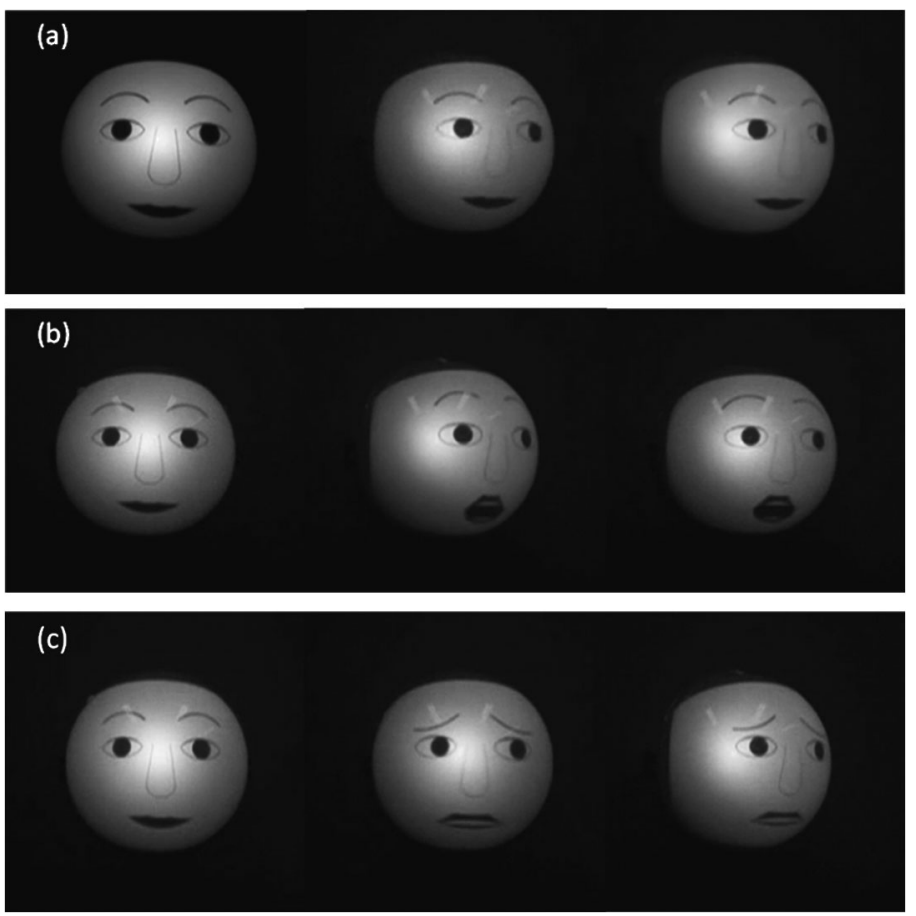

Figure 9. Examples of gaze movement with emotional expression of Kamin-FA1: (a) normal, (b) emotional expression of surprise and (c) fear.
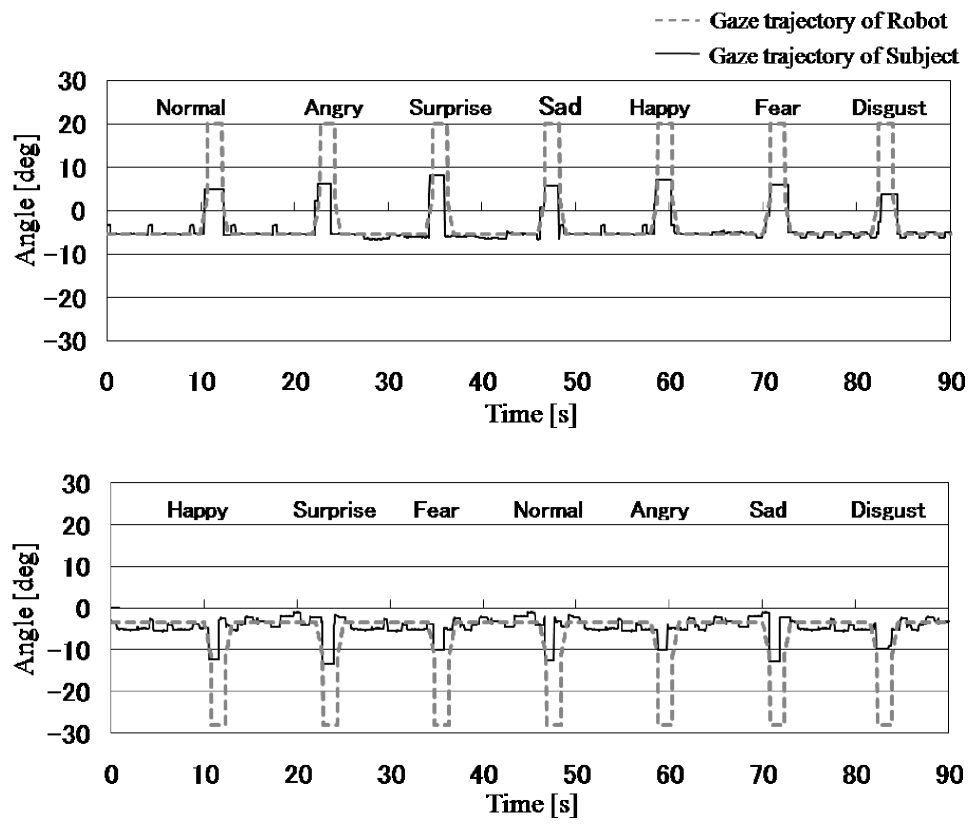

Figure 10. Examples of the gaze transition during the gaze guidance with emotional expression. The solid line is the gaze trajectory of the subject. The broken line is the gaze trajectory of Kamin-FA1. 
- - Gaze trajectory of Robot

- Gaze trajectory of Subject

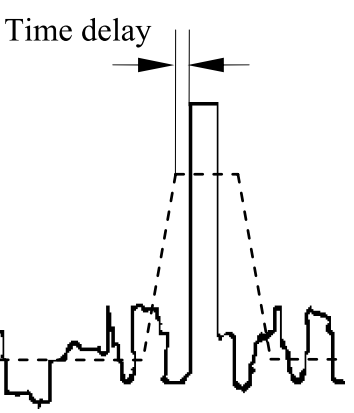

Figure 11. Maximum gaze angle and time delay. The solid line is the gaze trajectory of the subject. The broken line is the gaze trajectory of Kamin-FA1.

Table 2.

Maximum gaze angle and delay time for each emotional expression

\begin{tabular}{llllllll}
\hline & Normal & Happy & Angry & Surprise & Sad & Fear & Disgust \\
\hline Gaze angle $\left({ }^{\circ}\right)$ & 7.46 & 8.36 & 8.74 & 9.15 & 8.50 & 8.05 & 7.66 \\
Delay time $(\mathrm{s})$ & 0.80 & 0.82 & 0.84 & 0.72 & 0.75 & 0.85 & 0.80 \\
\hline
\end{tabular}

potential danger of the situation, then the subject will follow more accurately and quickly along the line of robotic sight to find the object or event.

Table 2 lists the average values among the 30 subjects for the maximum gaze angle and the delay time. The percentages of the average values calculated by (3) are shown in Figs 12 and 13. We used the average values during the 'rest' phase as the initial direction. The percentage value, $\gamma_{i}$, of emotional expression $i$ is calculated by the following equation using the average value of the normal expression $\beta$ :

$$
\gamma_{i}=\frac{\alpha_{i}-\beta}{\beta} \times 100(\%),
$$

where $\alpha_{i}$ is the average value of emotional expression $i$. The magnitude of the error bar is the standard error of the mean. Based on the results, it is shown that the gaze guidance performed by emotional motion is more effective than that without emotional motion. Furthermore, in the case of surprise emotion the maximum gaze angle is the largest and the delay time is the shortest among the emotional motions. Therefore, the surprise emotional motion is the most effective in gaze guidance when compared with other emotional motions. We tested the significant difference between the surprise emotion and the other emotions using the $f$-test. Since the number of comparisons is 5 , the value of the significance level is determined by 


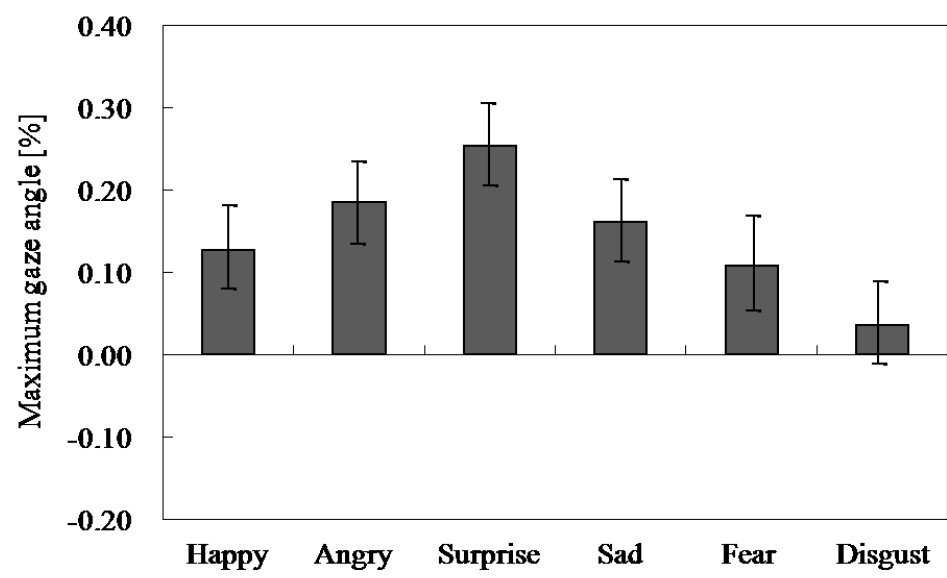

Figure 12. Human maximum gaze angle of gaze guidance.

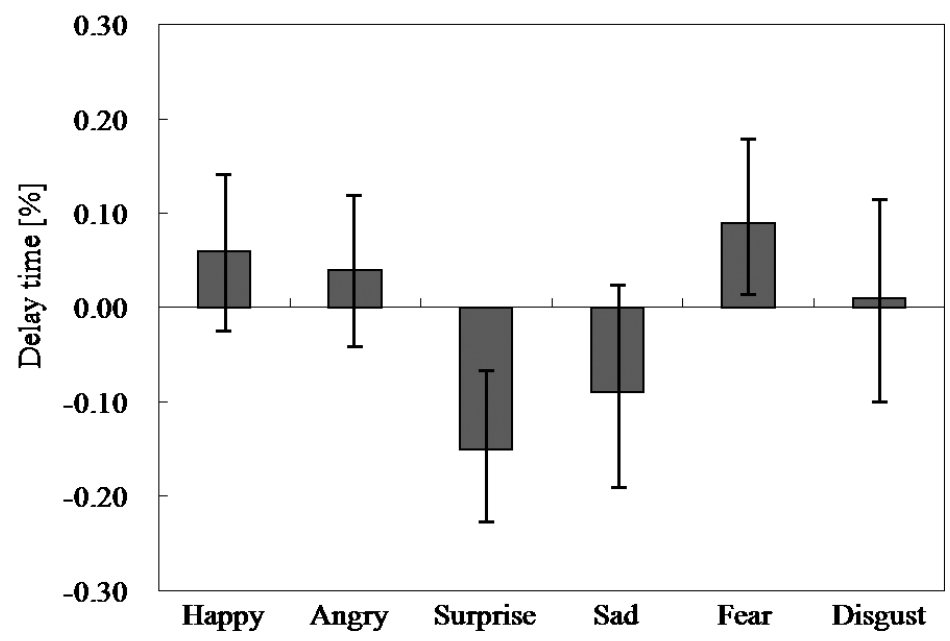

Figure 13. Delay of human gaze movement in gaze guidance.

0.01. In this sense, we found a significant difference between the surprise and normal emotions, and between the surprise and disgust emotions.

The results described above are consistent with the findings of Refs $[6,7]$ in terms of the presence of interactions between the gaze cuing and the emotional expressions. However, our result is not the same as that of Ref. [6] in terms of the kinds of emotion. The fear expression induced almost same the gaze cuing as that of the happy expression in this result.

\section{Reaction Speed of Operation}

We conducted experiments to investigate the reaction speed of operation in gaze guidance. When we recognize a dangerous situation, such as a pedestrian appear- 


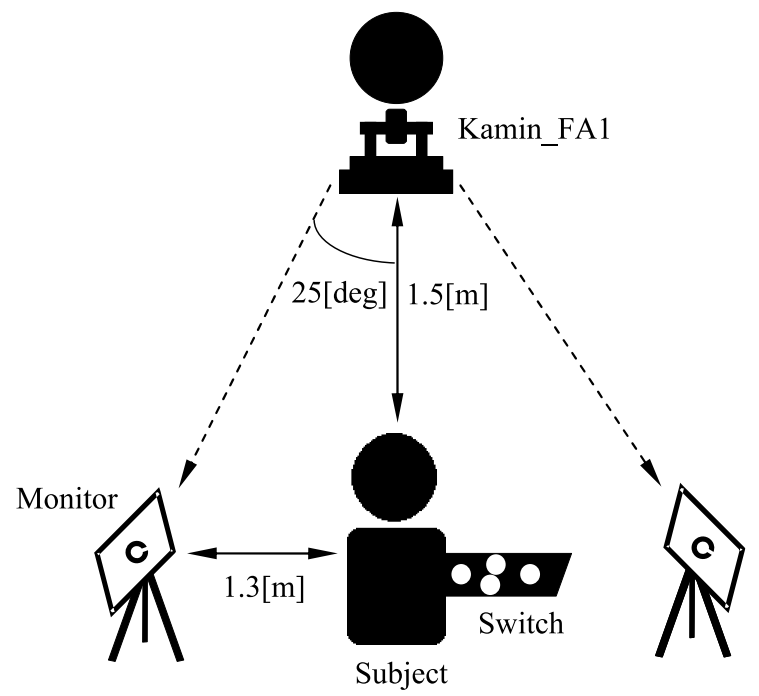

Figure 14. Schematic drawing of the reaction time experiment.

ing in front of a car, we need some response, e.g., applying the brake, as quickly as possible to avoid an accident. Therefore, the reaction speed of the response is important for safety. We evaluate the effect of emotional expression during gaze guidance in terms of the reaction speed of operation. The subject watches the target following the gaze guidance and recognizes the situation at the target. Then the subject performs an operation according to the recognition of the situation. We measure the reaction time from the beginning of gaze guidance to the beginning of the operation and designate this as the reaction speed of the operation.

Figure 14 shows the experimental setup of the reaction time experiment which includes Kamin-FA1, two monitors with a Landolt ring and a switch box. The subject sits down in front of Kamin-FA1 robot. When the robot's gaze with emotional expression changes from the subject position to one of the monitors, the subject watches the monitor following the gaze guidance and then the subject recognizes a Landolt ring of $20 \times 20 \mathrm{~mm}$ shown on the monitor. After the subject recognizes the direction of the Landolt ring, the subject pushes a switch, which corresponds to the direction of the Landolt ring. We measured the period from the beginning of gaze guidance to the beginning of the switch operation to examine the reaction time based on the emotional expression. The direction of the Landolt ring is changed after the start of gaze guidance. Figure 15 shows the Landolt ring displayed on the monitor (VGN-UX71, Sony) and the switch box. The gaze guidance motion of Kamin-FA1 is the same as that of the experiment in Section 3. The subjects were 10 students. We measured the data including the head angle and the pushing operation by a PC. The sampling time is $1 \mathrm{~ms}$.

Figure $16 \mathrm{a}$ and $16 \mathrm{~b}$ shows the trajectories of the robotic and subject's gazes, and the switch signal for the normal and the surprise expressions, respectively. These 


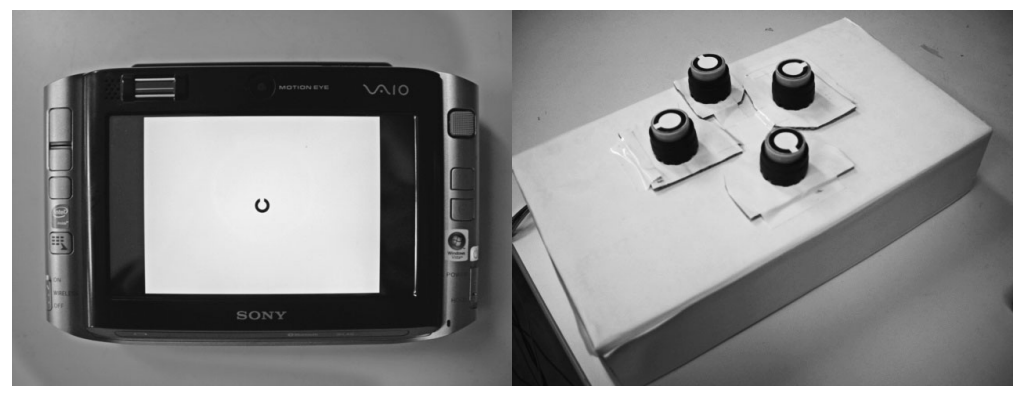

(a)

(b)

Figure 15. (a) Monitor (VGN-UX71) and (b) switch box.
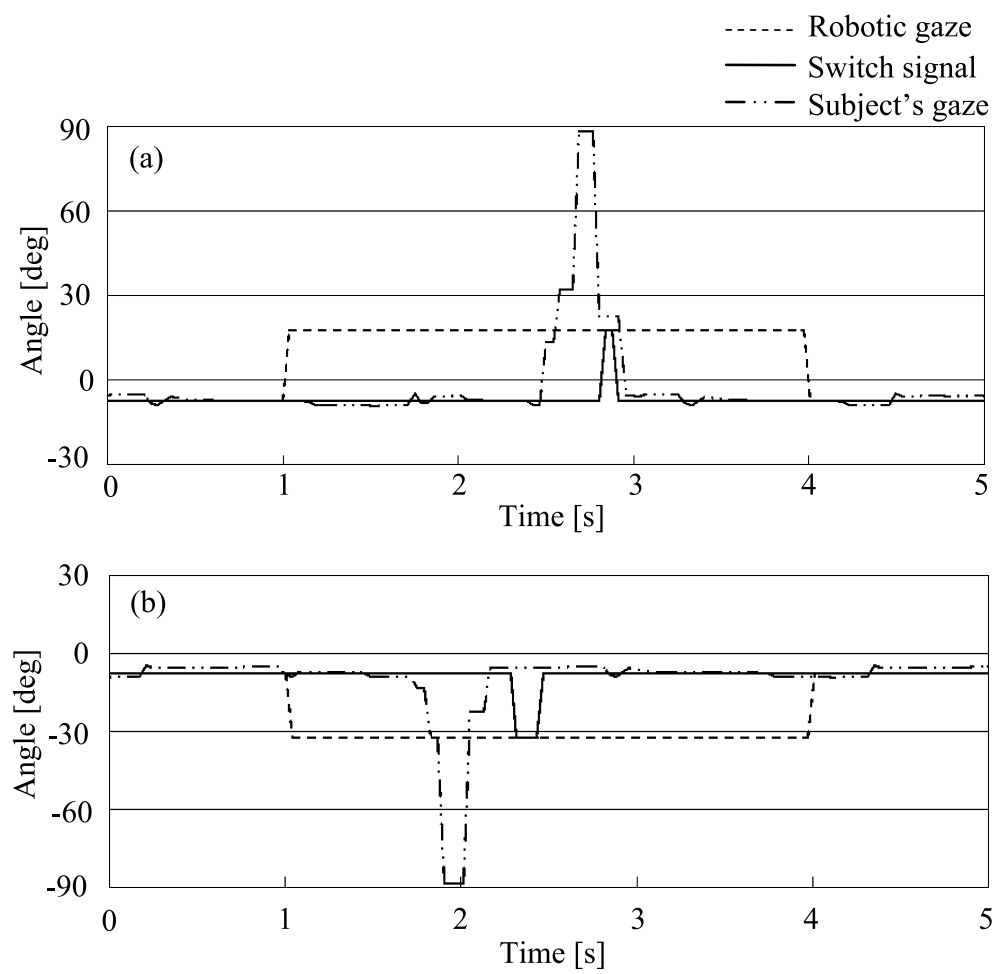

Figure 16. Examples of trajectories of robotic gaze, subject's gaze and switch signal. (a) Result of normal expression. (b) Result of surprise expression. The dotted line shows the robotic gaze. The solid line shows the switch signal. The double dashed line shows the subject's gaze.

figures show the examples of the trajectories of the subjects. The reaction time for each emotional expression is listed in Table 3. The percentage of the reaction time is calculated by (1). The difference between the reaction time of each emotional expression and the normal expression is shown as a percentage in Fig. 17. The percentage of the reaction time is the average value among the subjects. This graph 
Table 3.

Reaction time for each emotional expression

\begin{tabular}{llllllll}
\hline & Normal & Happy & Angry & Surprise & Sad & Fear & Disgust \\
\hline Reaction time (s) & 2.68 & 2.36 & 2.40 & 2.01 & 2.38 & 2.32 & 2.26 \\
\hline
\end{tabular}

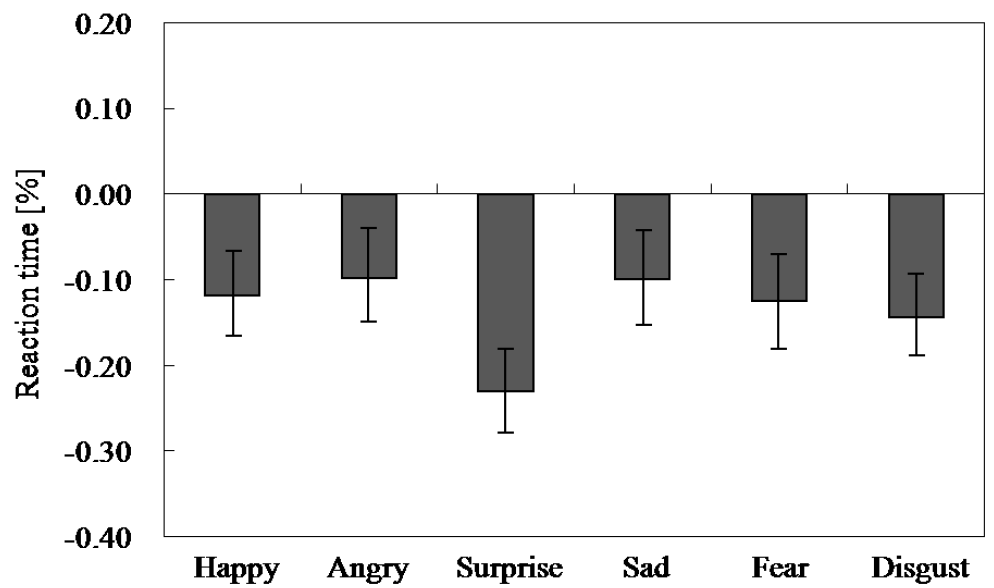

Figure 17. Reaction time of the operation in gaze guidance with emotional expression.

shows that the reaction time with the emotional expressions is shorter than that with the normal expression. There are significant differences among the emotional expressions. In the case of the surprise expression, the reaction time is shorter than for the other emotional expressions. Therefore, gaze guidance with surprise emotional expression causes faster reaction to the gaze guidance than with other emotional expressions.

\section{Situation Recognition}

We investigated the subject's impression of emotional expressions in terms of 'safety' or 'danger'. Subjects answered the impression at the target position on a sevenpoint scoring scale after gaze guidance with emotional expression. The conditions of gaze guidance are the same as the experiments in Section 3. The subjects were 30 students.

Figure 18 shows the results of this experiment. There are significant differences between the surprise and normal emotions, and between the surprise and happy emotions. First, in the case of anger, surprise, sad, fear and disgust, the subjects were alerted to a potentially dangerous situation requiring their attention at the gaze target. In the case of normal and happy, the subjects were assured of a safe situation at the gaze target. The emotional expressions of angry and happy resulted in the highest scores of each impression. The emotional expression of surprise 


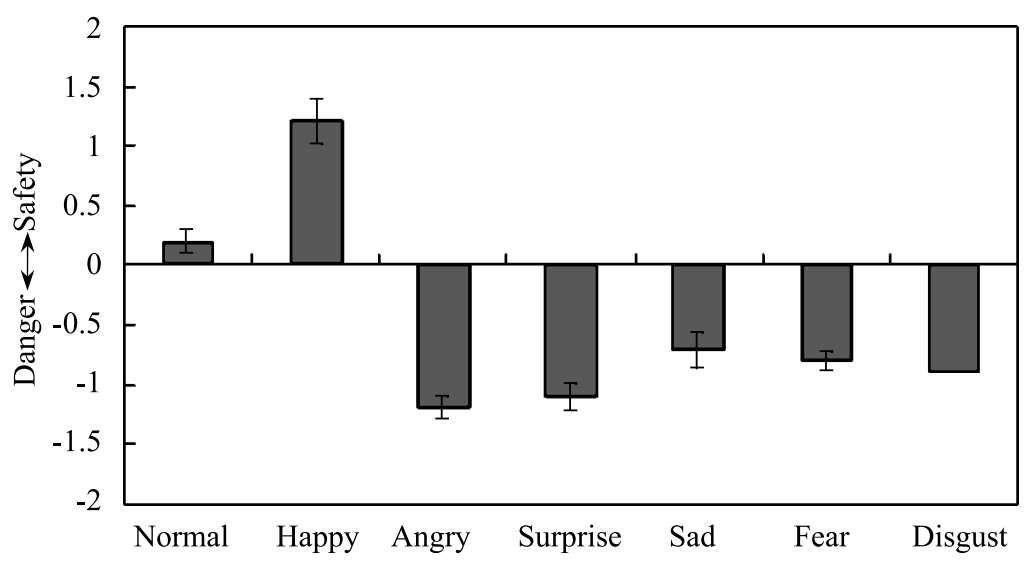

Figure 18. Results of psychological experiments.

also worked in alerting the subjects to a potentially dangerous situation. Therefore, the emotional expressions of angry and surprise are effective in representing the impression of 'danger', and the emotional expression of happy is effective in representing the impression of 'safety'.

\section{Conclusions}

We have investigated gaze guidance using a head robot Kamin-FA1. We used not only gaze control of the robot, but also the facial expression to guide human gaze to the target. We focused this study on the effect of the emotional expression for gaze guidance and reaction speed in gaze guidance. Also, the differences among the emotional expressions in terms of the effectiveness of gaze guidance and representing the situation at the target of the gaze were investigated.

It was found that gaze guidance combined with emotional expression was more effective than that without emotional expression. Furthermore, in the case of surprise emotion, the maximum gaze angle was larger and the delay time was shorter than those of the normal expression. Therefore, surprise is a more effective emotional expression in gaze guidance compared with the normal expression. Emotional expressions of anger and surprise alerted subjects to a potentially dangerous situation, and the emotional expression of happy conveyed to the subjects a safe situation at the target of gaze. The results are consistent with the findings of Refs $[6,7]$ in terms of the presence of interactions between the gaze cuing and emotional expressions.

This technique has potential use for a car interface to guide the driver's gaze in order to produce a safer driving environment. In future studies, we think it is important to determine the design method of the emotional expression of the robot for accurate and quick gaze guidance. 
Acknowledgements

We are grateful to Assistant Professor Michael Honywood of Shinshu University who provided critical assistance on our manuscript.

\section{References}

1. C. L. Breazeal, Designing Sociable Robots. MIT Press, Cambridge, MA (2002).

2. H. Miwa, K. Itoh, M. Matsumoto, M. Zecca, H. Takanobu, S. Roccella, M. C. Carozza, P. Dario and A. Takanishi, Effective emotional expressions with emotion expression humanoid robot WE-4RII, in: Proc. IEEE/RSJ Int. Conf. on Intelligent Robot and Systems, Sendai, pp. 2203-2208 (2004).

3. M. Hashimoto and D. Morooka, Robotic facial expression using a curved surface display, J. Robotics Mechatron. 18, 504-510 (2006).

4. M. Hashimoto, M. Yamano and T. Usui, Effects of emotional synchronization in human-robot KANSEI communications, in: Proc. 18th IEEE Int. Symp. on Robot and Human Interactive Communication, Toyama, in press (2009).

5. T. Mitamura, K. Ohta, H. Hozui, M. Inoue, A. Sakai and J. Osada, Development of robotic agent on Nissan Pivo 2, in: Proc. Human Interface Symp., Osaka, pp. 1129-1132 (2008).

6. P. Putman, E. Hermans and J. van-Honk, Anxiety meets fear in perception of dynamic expressive gaze, Emotion 6, pp. 94-102 (2006).

7. D. Sander, D. Grandjean, S. Kaiser, T. Wehrle and K. R. Scherer, Interaction effects of perceived gaze direction and dynamic facial expression: evidence for appraisal theories of emotion, Eur. $J$. Cognit. Psychol. 19, 470-480 (2007).

8. J. K. Hietanen and J. M. Leppänen, Does facial expression affect attention orienting by gaze direction cues? J. Exp. Psychol. Hum. Percept. Perform. 29, 1228-1243 (2003).

9. A. Mathews, E. Fox, J. Yiend and A. Calder, The face of fear: effects of eye gaze and emotion on visual attention, Vis. Cognit. 10, 823-835 (2003).

10. J. Tipples, Fear and fearfulness potentiate automatic orienting to eye gaze, Cognit. Emotion 20, 309-320 (2006).

11. T. Nagai, K. Hosoda, A. Morita and M. Asada, Emergence of joint attention through bootstrap learning based on the mechanisms of visual attention and learning with self-evaluation, Trans. Japan. Soc. Artif. Intell. 19, 10-19 (2004).

12. Y. Yoshikawa, K. Shinozawa, H. Ishiguro, N. Hagita and T. Miyamoto, The effect of responsive eye movement and blinking behavior in a communication robot, in: Proc. IEEE/RSJ Int. Conf. on Intelligent Robots, and Systems, Beijing, pp. 4564-4569 (2006).

13. H. Osawa, J. Mukai and M. Imai, Anthropomorphized object using displaying robot, in: Proc. 20th Ann. Conf. of the Japanese Society for Artificial Intelligence, Tokyo, 3F3-2, pp. 1-4 (2006).

14. P. Ekman and W. V. Friesen, Facial Action Coding Consulting. Psychologist Press, New York, NY (1977). 


\section{About the Authors}

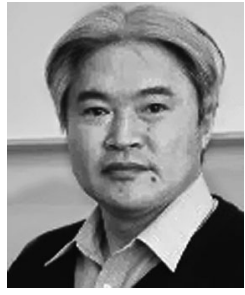

Minoru Hashimoto received the MS and $\mathrm{PhD}$ degrees from the University of Tokyo, in 1980 and 1983, respectively. From 1982 to 1988, he was a Research Associate of the Robotics Laboratory in the Department of Mechanical Engineering, University of Electro-communications. From 1988 to 1998, he was an Associate Professor of the Department of Mechanical Engineering, Kagoshima University. Since 1999, he has been a Professor of Department of the Kansei Engineering, Shinshu University. His research interests are communication robots, motion assist systems and artificial muscles.

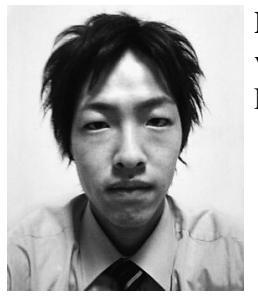

Hiromi Kondo received the BS degree in Kansei Engineering from Shinshu University, in 2007. Since 2007 he has been an Engineer at Hayashi Telempu Co. Ltd.

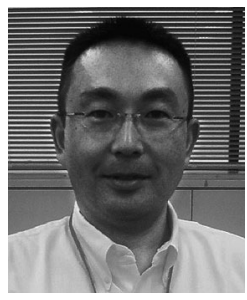

Yukimasa Tamatsu received the BS degree in Control Engineering from Kyushu Institute of Technology, in 1987. Since 1987, he has been an Engineer at DENSO Co. Ltd. After he engaged in the development of a car control system, he has been engaged in the development of a sensing system of moving environments. 